MS22 Beyond multipolar refinement

Chairs: Alessandro Genoni, Simon Grabowsky

\section{MS22-01 Quasi-harmonic Treatment of Thermal Effects on Electron Charge and Momentum Densities of Solids from $A b$ initio Calculations}

\author{
Alessandro Erba \\ 1. Chemistry Department, University of Torino, via Giuria 5, \\ 10125 Torino (Italy) \\ email: alessandro_erba@virgilio.it
}

The inclusion of thermal effects on computed properties of solids from $a b$ initio calculations still represents a challenge to state-of-the-art quantum-chemical methods, particularly so when the limitations of the harmonic approximation (HA) to the lattice potential have to be overcome. When the HA is used, indeed, the vibrational contribution to the free energy of the crystal is assumed to be independent of volume. As a consequence, a variety of physical properties would be wrongly described [1]: null thermal expansion, elastic constants independent of temperature as well as the bulk modulus, equality of constant-pressure and constant-volume specific heats, infinite thermal conductivity as well as phonon lifetimes, etc.

If the explicit calculation of anharmonic phonon-phonon interaction coefficients remains a rather computationally demanding task, with implementations often limited to a molecular, non-periodic context, a simpler, though effective, approach for correcting most of the above mentioned deficiencies of the HA is represented by the so-called quasi-harmonic approximation (QHA) [2].

In this contribution, I discuss a series of $a b$ initio techniques, as they have been implemented into the CRYSTAL program, for the inclusion of thermal effects on several (structural, elastic, thermodynamic and electronic) properties of solids beyond the HA, by explicitly accounting for thermal lattice expansion [3-6]. In particular, the QHA is used to study thermal effects on the EMD of crystalline LiF [7]. Preliminary results are also presented of a study where the QHA is applied to compute atomic anisotropic displacement parameters (ADPs) and dynamic X-ray structure factors of the urea molecular crystal, from dispersion-corrected lattice dynamical ab initio calculations [8].

\section{References}

[1] N. W. Ashcroft and N. D. Mermin, Solid State Physics, Saunders College, Philadelphia, USA (1976).

[2] R. E. Allen and F. W. De Wette, Phys. Rev., 179, 873-886 (1969).

[3] A. Erba, J. Chem. Phys., 141, 124115 (2014)
[4] A. Erba, M. Shahrokhi, R. Moradian and R. Dovesi, J. Chem. Phys., 142, 044114 (2015)

[5] A. Erba, J. Maul, R. Demichelis and R. Dovesi, Phys. Chem. Chem. Phys., 17, 11670-11677 (2015)

[6] A. Erba, J. Maul, M. De la Pierre and R. Dovesi, J. Chem. Phys., 142, 204502 (2015)

[7] A. Erba, J. Maul, M. Itou, R. Dovesi and Y. Sakurai, Phys. Rev. Lett., 115, 117402 (2015)

[8] A. Erba, J. Maul and B. Civalleri, Chem. Commun., 52, 1820-1823 (2016)

Keywords: thermal effects, quasi-harmonic approximation, ab initio calculations, Compton profiles, ADPs 